\title{
Cell Cycle Kinetics
}

National Cancer Institute

\section{Source}

National Cancer Institute. Cell Cycle Kinetics. NCI Thesaurus. Code C18491.

Cell cycle kinetics is the rate at which a cell progresses through the cell cycle. It is a general indication of how fast a tissue or cell sample is proliferating. This rate is generally increased in more aggressive tumors and can be measured by various biochemical methods such as the incorporation of labeled nucleotides into replicating DNA or the level of expression of cell cycle proteins or other proliferation markers. 